\title{
Modeling of Agricultural Spatial Objects with Heterogeneous Dynamically Changing Spatial Structure
}

\author{
R V Brezhnev ${ }^{1}$, Yu A Maglinets ${ }^{1}$, K V Raevich ${ }^{1}$ and G M Tsibulski ${ }^{1}$ \\ ${ }^{1}$ FSAEI All-Russia Association of Siberian Federal University, Institute of Space and \\ Information Technologies, Kirenskogo str. 26, Krasnoyarsk, Russia, 660074
}

\begin{abstract}
This article provides research for the models describing the spatial object of Agricultural Land (AL) as observed at medium- and high-spatial resolution satellite images. This object is characterized by variable reflectance features during the vegetation season. These variations are caused both by natural and man-induced environmental effects and by soil cover properties. The vegetation cover growth rates may vary at different areas within the agricultural contour which leads to the creation and development of heterogeneities. The research focuses on the spatial modeling of the agricultural object with heterogeneous dynamically changing spatial structure. A model which allows matching the values of parameters measured at space images with reference and abnormal object behavior has been developed. This model describes the object of research on the level of geometric and relational structures, thereby making it possible to determine spectral and metrical features as part of image heterogeneities, find interrelations between these features within the time limits and map these heterogeneities. The results obtained were tested when monitoring the state and development trends for grain crops at the tested objects in the Sukhobuzimsky district of the Krasnoyarsk Territory.
\end{abstract}

\section{Introduction}

The remote monitoring methods are used in agricultural sector to solve a wide range of issue-related tasks, including the assessment of state and utilization of crop rotation fields, soil fertility and soil degradation parameters, state of vegetation cover at plough lands, deposits, hay and grazing lands, forecasting of crop yield and etc. The objects of agricultural monitoring are characterized by variations in time. Variations observed at space images of such objects are related to the natural vegetation stages (change of phenological phases), climatic conditions, and agrotechnical measures (ATM) performed. The type of variations is determined by the properties of soil cover, fertilizers applied and some other factors specified in [1-2]. In some cases, a change in spatial structure of agricultural land is also observed in the form of uneven crop growth within the object contour. Thus, this type of the monitored objects is referred to spatial objects with dynamically variable structure.

General principles for the use of spatial-temporal aspects of dynamically variable spatial objects have been so far put together in the works of B. Claramunt, M. Molenaar, R. Nandal, T. Ott, D. Peuquet, A.Renolen, M. Yuan, Yu. K. Korolev, V.S. Tikunov and etc [3-5]. Event-oriented, 3-domain and History Graph models and etc have been developed. However, these models are aimed at presentation of objects with time-variable boundaries whereas handling of objects with dynamically variable structure is under-developed in the literature. 
The object of the present research is a spatial object being vegetable agricultural land with a limited agricultural contour namely the agricultural site [6], further hereon AL or field. This spatial object may be matched with an attribute vector: spectral, textural, metric, topological and etc. During the vegetation season the values of the observed parameters' vector may vary and some attributes may be dynamically added to the formed description as being exhibited at a certain period of time. Furthermore, structural heterogeneity of the object should be taken into account when modeling, moreover the type of heterogeneity is also time variable.

Detection of local heterogeneities of the object and their variation in time at space images allows assessing the uniformity of crop germination capacity, their degradation as well as the performance rate of the planned agrotechnical activities which, in particular, provides for precision farming.

\section{Conceptual Model of Agricultural Land Spatial Object}

The object of research is characterized by the following types of parameters which are essential for the arrangement of a monitoring system [7]:

1. Spatial confinement (exact geographical references of the object with due account of topological parameters).

2. Name and breed of crop for the current field season.

3. Seasonal vegetation development schedule in the form of changing crop phenological stages.

4. Possible abnormalities (in connection with phenological stages) such as non-germination, soil overwatering, drought, impurities and etc.

5. Man-induced impact as a result of planned and performed agrotechnical activities.

6. Crop characteristics (such as integral state, biomass, leaf surface and etc.).

The abovementioned parameters may be detailed for the specific natural and climatic states and type of vegetation. Thus, Figure 1 shows a set of allowable states of cereal crop, in particular spring wheat, barley and oat correlated with the time intervals $(T m)$ as determined for crop rotation in central regions of the Krasnoyarsk Territory.

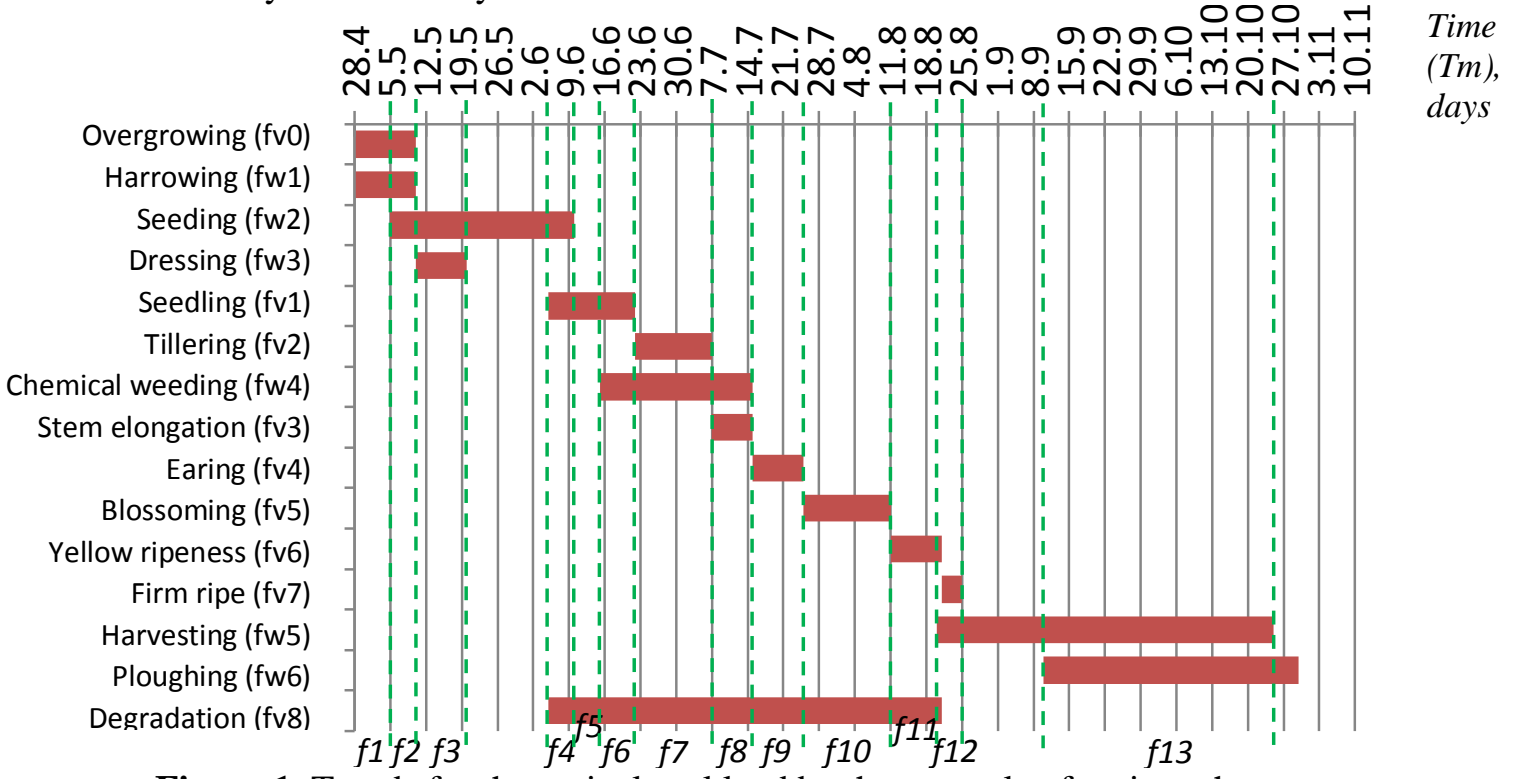

Figure 1. Trends for the agricultural land by the example of spring wheat.

With account of the performed agrotechnical activities the object may be in one of the following $f^{w i}$ states: $f^{w 1}$ - harrowing, $f^{w 2}$ - seeding, $f^{w 3}$ - dressing, $f^{w 4}-$ chemical weeding, $f^{w 5}-$ harvesting, $f^{w 6}$ - ploughing. The crop may also be in certain $f^{v i}$ states which are determined by phenological stages of a certain time sequence. Thus, stages for the cereal crops researched herein describe the following states of the object: $f^{v 0}$ - overgrowing, $f^{v 1}$ - germination, $f^{v 2}$ - tillering, $f^{v 3}$ - stem elongation, $f^{v 4}$ - earing, $f^{v 5}$ - blossoming, $f^{v 6}$ - yellow ripeness, $f^{v 7}$ - firm ripe stage. A cereal crop may pass to $f^{v 8}$ state that is degradation at each stage of vegetation which indicates the 
abnormal crop development. When in standard state, the object is presented either by open soil or homogeneous vegetable carpet corresponding to a certain development stage.

Interception of standard time ranges for the object being in one or another state results in heterogeneous structure of the object and requires consideration of the object's composite states which can be analyzed on State-Transition Diagram $G$ (Figure 2):

where $F$ is a variety of the object's states:

$$
G=(F, R) \text {, }
$$

$R$ - total state transitions:

$$
F=f^{v i} \cup f^{w j}
$$

$$
R=r^{1} \cup r^{2} \cup r^{3},
$$

where $r^{1}$ is a variety of operators describing a crop development in time (natural change of phenological phases), $r^{2}$ is a variety of operators describing abnormal crop development, $r^{3}$ is a variety of operators modeling the performance of agrotechnical activities.

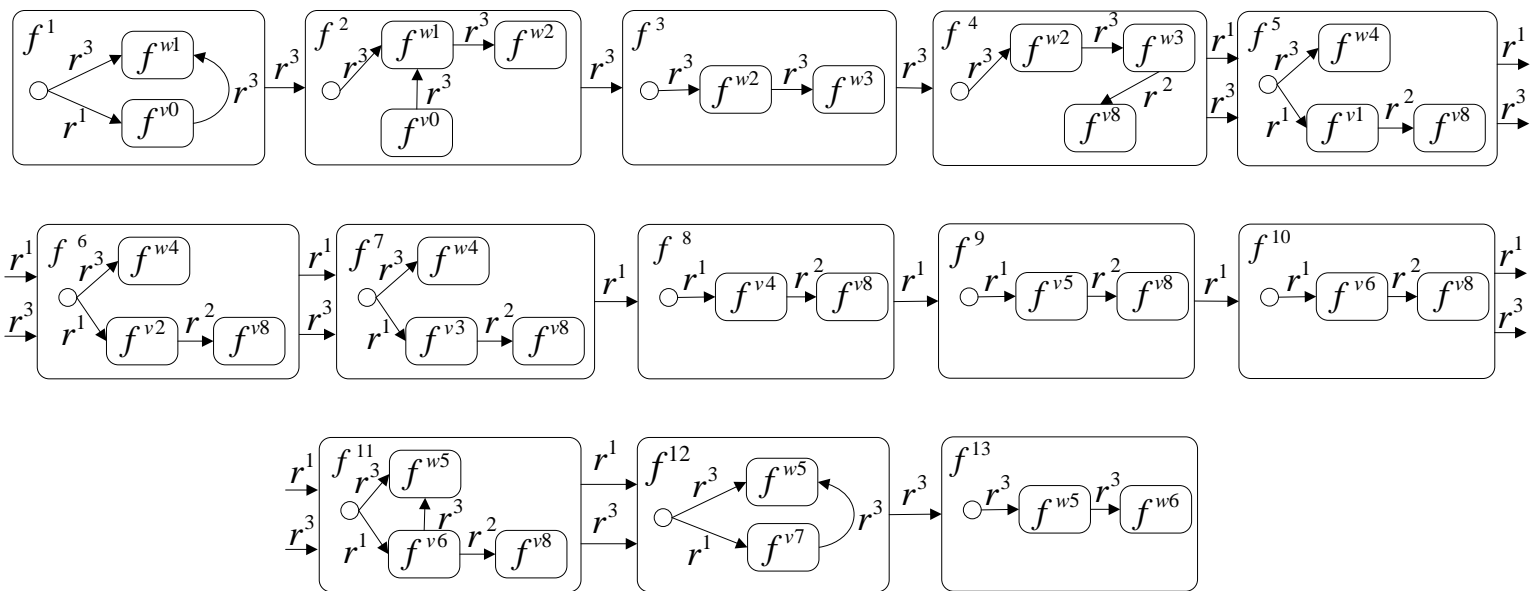

Figure 2. Dynamic model of agricultural land spatial object.

\section{Model of a Spatial Object with Heterogeneous Dynamically Variable Structure}

The changes in the object's structure have been traced and interpreted with the help of Earth Remote Sensing (ERS). Satellite images (Figure 3) show heterogeneities as a plurality of segments which may be interpreted by spectral characteristics.

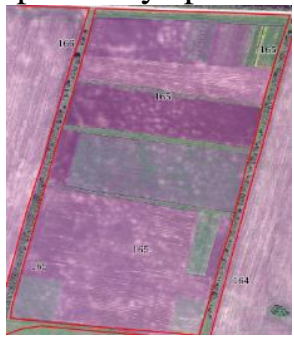

a

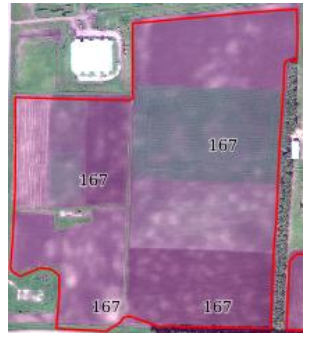

$\mathrm{b}$

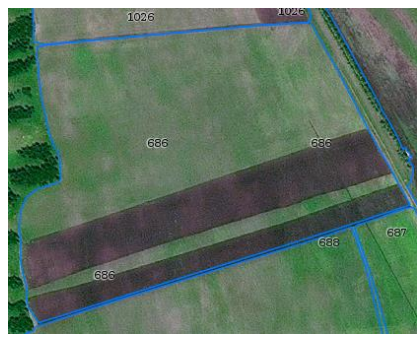

c

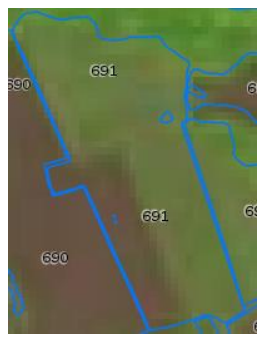

d

Figure 3. Heterogeneities within the contours: $a, b-\operatorname{Nos} 165,167$ as per the highresolution data WorldView-2 (0.5 m); c - No 686 as per the high-resolution data Spot-6 (6 m); - No 691 as per the medium-resolution data Landsat-8 (30 m).

Each state is characterized by a set of features which are essential for setting the monitoring task by an end user as well as for allocation and interpretation of states of the object's heterogeneities. The main categories of such features are given below.

1. Metrical features including:

1.1. Geographical coordinates of the object in the set projection $P_{\Pi}=\left\{\left(x_{i}, y_{i}\right)\right\}$, specifying its location. Surface area of polygonal-shaped object calculated as follows: 


$$
N S=\frac{1}{2}\left|\sum_{i=1}^{n-1} x_{i} y_{i+1}+x_{n} y_{1}-\sum_{i=1}^{n-1} x_{i+1} y_{i}-x_{1} y_{n}\right|
$$

where $\left(x_{i}, y_{i}\right), i=1,2, \ldots, n$ are a sequence of coordinates of the neighboring vertexes of polygon, $\left(x_{i+1}, y_{i+1}\right)=\left(x_{1}, y_{1}\right)$.

Perimeter of the object:

$$
N P=\sum_{i=1}^{n} \sqrt{\left(x_{i+1}-x_{i}\right)^{2}+\left(y_{i+1}-y_{i}\right)^{2}},
$$

where $i=1,2, \ldots, n$ is a number of the contour vertexes.

Thickness of the object:

$$
T=4 \pi\left(N S / N P^{2}\right) \text {. }
$$

2. Spectral features describe the object in various spectral ranges.

Normalized Difference Vegetation Index (NDVI) has shown good results in Agricultural Land structure classification [8]:

$$
N D V I=(N I R-R E D) /(N I R+R E D),
$$

where $N I R$ is a value of spectral channel corresponding to the near IR range with the wavelength of $0.76-1 \mu \mathrm{m} ; R E D$ is a value of spectral channel corresponding to the visible-light spectrum with the wavelength of $0.6-0.76 \mu \mathrm{m}$ (red light). When modeling, this Index was compared to other vegetation indexes such as DVI (Richardson and Everitt, 1992), RVI (Jordan, 1969), WDVI (Clevers, 1988), SAVI (Huete, 1988) and etc. with the help of experimental evidence. The above spectral indexes as well as the eigenvalues of NIR and RED spectral channels are informative for vegetation analysis. The developed model is invariant with respect to the choice of a specific index.

3. Time features $(\mathrm{Tm})$ specify discrete variations of the object's states.

The image of an object shall be transferred from the iconic level to the level of geometrical and relational structures in order to solve the tasks of heterogeneities' allocation at satellite images and their presentation in the form of heterogeneities' maps as well as to provide information support to the end user. This shall allow calculation of spectral and metrical features of heterogeneities and identification of interrelations between these features and elements of $F$ plurality within the set time ranges.

The resulting model of an object with a heterogeneous dynamically changing structure can be presented at the level of geometrical and relational structures as follows:

$$
\text { Agricultural Land }=<I D, P_{\Pi}, N S, N P, T, N, T m, K, E, \varphi, F>\text {, }
$$

where $I D$ is an identifier (field number), $P_{\Pi}$ is a plurality of the object's coordinates: $P_{\Pi}=$ $\left\{\left(x_{1}, y_{1}\right),\left(x_{2}, y_{2}\right), \ldots,\left(x_{n}, y_{n}\right)\right\}$, where $x, y$ are latitude/longitude point coordinates accordingly, $N S$ surface area, $N P$ - perimeter, $T$ - thickness, $N$ - plurality of $N D V I$ value ranges corresponding to the specific assessment of the object state, $T m$ - plurality of time ranges for the object state transitions.

In addition to the above features, various semantic data related to the object and specifying the peculiarities of the object development in time must be borne in mind for the object allocation by the end user when setting the monitoring tasks. It includes: set agricultural crop $K=\left\{k^{i}\right\}$ with its own reference model of development in time which is determined, on one hand, by agrotechnical activities identified as a plurality of time-scheduled events $E=\left\{e^{i}\right\}$ and influencing transition of the object state and, on the other hand, by successive phenological development stages $\varphi=\left\{\varphi^{i}\right\}$ identifying the plurality of the object states $F(2)$.

The expression (8) describes Agricultural Land object at the level of geometrical and relational structures and allows identification of spectral and metrical features within the localized contours of heterogeneities, setting interrelation of these features in preset time ranges as well as presentation of findings in the form of heterogeneities' map.

\section{Experimental Approval}

Free satellite images Landsat- 8 and Sentinel-2A (VNIR shooting mode) were selected as input data for the experimental localization of heterogeneities. Spectral channels in red (RED) and near IR (NIR) 
ranges with wavelengths of $0.63-0.68$ and $0.78-0.89 \mu \mathrm{m}$ accordingly were used. Fourteen scenes were obtained in 2016 and ten scenes in 2017.

The test area within Minderlinskoe educational-research farm in Sukhobuzimsky district of Krasnoyarsk Territory (Figure 4) was chosen for experimental research of the spatial object heterogeneous structure. The chosen area is located in the central part of the Krasnoyarsk Territory. It stretches for $140 \mathrm{~km}$ from West to East and for almost $100 \mathrm{~km}$ from North to South. The total surface area is 561,260 ha $\left(5,613 \mathrm{~km}^{2}\right)$. Forest-steppe landscape in the western part grades into taiga area on the right bank of the Yenisei River. The survey area the agricultural fields are located at have the following references: between $92.5^{\circ} \mathrm{E}$ and $94.7^{\circ} \mathrm{E}$ and between $56.2^{\circ} \mathrm{N}$ and $56.9^{\circ} \mathrm{N}$.

The routine of experiment includes atmospheric correction of RED and NIR channels by DOS method in order to obtain correct values when calculating Normalized Difference Vegetation Index (7); the threshold segmentation by the obtained NDVI channel; correction of the obtained segments to the level of geometry structures into the format of SHP ESRI vector layer and layer importing into PostgreSQL DBMS for relational presentation of the features of heterogeneities; calculation of model characteristics (4-6) and averaged NDVI value for each segment.

Allocation of heterogeneous areas of Agricultural Land and interpretation of their states shall be considered as the task of the satellite image segmentation by the spectral NDVI feature in accordance with the homogeneity criteria which are true for the test objects under consideration with cereal crops at the researched area and which are defined as follows:

$$
s(x, y)=\left\{\begin{array}{lr}
\lambda_{0}=\text { background } & \text { with }-1 \leq f(x, y)<0,025, \\
\lambda_{1}=f^{w 1} \text { or } f^{w 6} & \text { with } 0,025 \leq f(x, y) \leq 0,26, \\
\lambda_{2}=f^{w 2} \text { or } f^{w 5} & \text { with } 0,26<f(x, y) \leq 0,43, \\
\lambda_{3}=f^{v 1} \text { or } f^{v 6} \text { or } f^{v 7} & \text { with } 0,43<f(x, y) \leq 0,57, \\
\lambda_{4}=f^{v 2} & \text { with } 0,57<f(x, y) \leq 0,65, \\
\lambda_{5}=f^{v 3} & \text { with } 0,65<f(x, y) \leq 0,72, \\
\lambda_{6}=f^{v 4} \text { or } f^{v 5} & \text { with } 0,072<f(x, y) \leq 0,85
\end{array}\right.
$$

where $s(x, y)$ is a segmented image, $\lambda_{0}, \lambda_{1}, \ldots, \lambda_{m-1}$ are marks of segments corresponding to the preset state, $f(x, y)$ is a function of the image brightness.

The threshold selection procedure during segmentation is based on analysis of statistical data collected starting from 2013 by field NDVI measurements and calculation of correlation dependence with spectral vegetation features as per Landsat-7,8, Sentinel-2A, WorldView-2, Spot-4,6 satellite data.

Therefore, the on-site field investigations of test fields were performed to verify the satellite measurements. The base map of test fields is shown in Figure 4. Four test fields were selected at the test range. These fields are cropped with one culture: with oat (176), barley (155) and wheat (157, 166) crops.

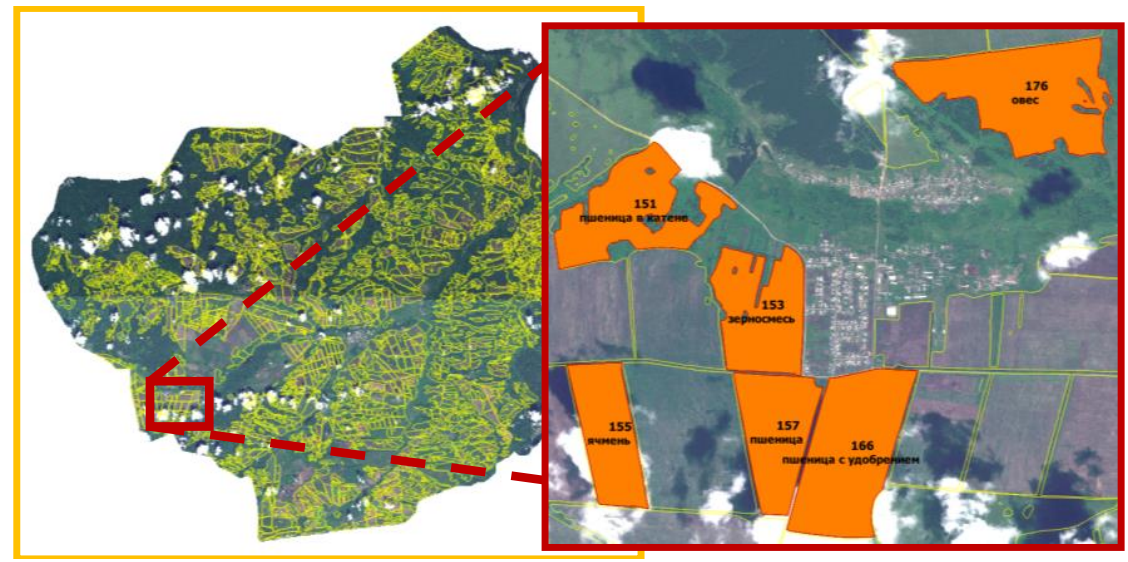

Figure 4. Base map of test fields. 
The FI-20A photometrical meter was used for NDVI measurement in order to eliminate atmospheric effects such as clouds or haze. This meter is meant for the day-time operation at the ambient air temperature from 5 to $40^{\circ} \mathrm{C}$ and relative air humidity up to $80 \%$ with no precipitation.

The resultant model allows revealing heterogeneities corresponding to the preset NS dimensions and $\mathrm{T}$ form at the geometrical level. This can be used in precision farming when analyzing spot tillage and calculating the required amount of crop protectors and etc. Therewith only segments which state does not correspond to the current phenological stage are taken into consideration. This is done by filtration of localized segments with due account of their possible pertinence $\tau_{F}$ to some actual state $f_{A}^{i} \in F$ and of comparison $f_{A}^{i}$ with the reference state $f_{\mathrm{H}}^{i}$ typical for that time.

Determination of state $F$ for heterogeneous area $w_{i}$ is the dependence function of time $T m$ and of the average vegetation index $\bar{N}: F_{w_{i}}(T m, \bar{N})$.

The segmented image is worked out to the level of object-relational structure where each area $w_{i} j$ zo of Agricultural Land object with dimensions $N S_{j}^{i}$ and form $T_{j}^{i}$ which satisfy the given values is space-outlined by a plurality of coordinates $\left\{P_{\Pi j}^{i}\right\}$, is fixed by the unique identifier $I D$ specifying the "whole-part" relation with the object itself and shows the actual state $f_{A j}^{i}$. This actual state may or may not correspond to the reference state $f_{\mathrm{H}}^{i}$ depending on the averaged vegetation index $\bar{N}_{j}^{i}$ and time $T m$. The reference state is determined by the actual phenological stage $\varphi_{j}^{i}$ or by the agrotechnical activity $e_{j}^{i}$ specific for the set crop $e_{j}^{i}$, this being presented in the object-relational model of Agricultural Land heterogeneity:

$$
3 C X H_{j}=<I D,\left\{P_{\Pi j}^{i}\right\}, N S_{j}^{i}, N P_{j}^{i}, T_{j}^{i}, \bar{N}_{j}^{i}, T m, k_{j}^{i}, e_{j}^{i}, \varphi_{j}^{i}, f_{A j}^{i}>.
$$

Segmentation results as per Sentinel-2A data dd. 05.06.2016 are shown in Figure 5-a, b and as per Landsat-8 data dd.15.06.2016 are shown in Figure 5-c, d.

a)
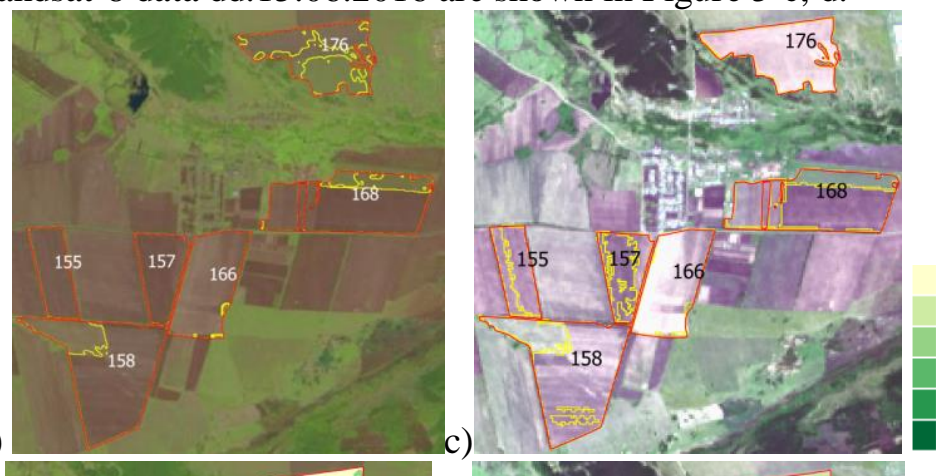

$0.025-0.26$ harrowing, plowin

$0.26-0.43$ seeding, harvesting

$0.43-0.57$ seedling and growing, yellow and firm ripeness

$0.57-0.65$ tillering

$0.65-0.72$ stem elongation

$0.72-0.85$ earing, blossoming
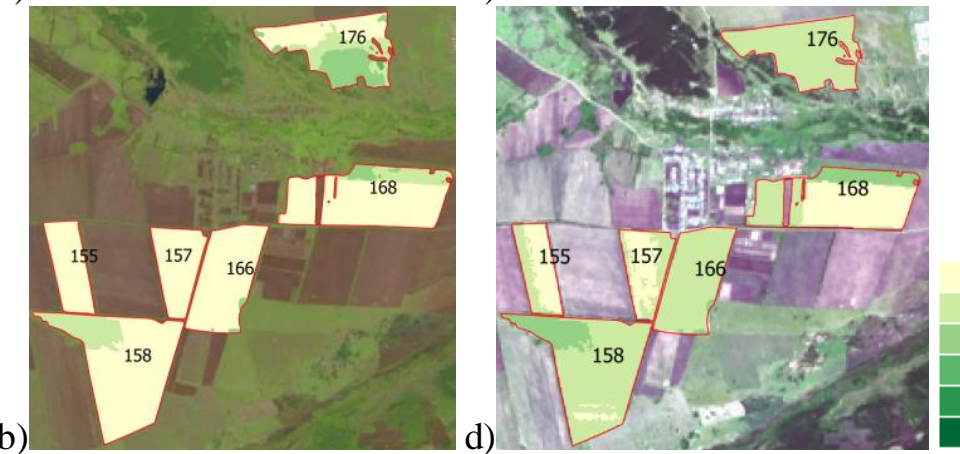

$0.26-0.43$ seeding, harvesting

$0.43-0.57$ seedling and growing, yellow and firm ripeness

$0.57-0.65$ tillering

$0.65-0.72$ stem elongation

$0.72-0.85$ earing, blossoming

Figure 5. Results of heterogeneities' allocation: a, b - Sentinel-2A dd. 05.06.2016;

c, d - Landsat-8 dd. 15.06.2016.

The results received on June 05,2016 , showed that the area of the objects under research was mainly presented by open soil. The situation changed in 10 days that is on June 15, 2016, with the volume of biomass increasing in accordance with the current phenological stage of the cereal crop 
development (the seedling stage) and the beginning of the tillering stage which is well shown by a change of the local objects' structure.

Correlation analysis of the spectral survey showed high (0.87-0.99) correlation with the results of measurements in the analyzed satellite images. Twenty to thirty measurements were taken to calculate the average NDVI value for each field. The space coordinates of the measurement points were recorded by GPS-receiver.

\section{Conclusion}

This article deals with the resultant model of Agricultural Land spatial object with dynamically changing spatial structure. This model allows:

- due consideration of heterogeneous spatial dynamically changing structure of the object;

- calculation of spectral and metrical features of heterogeneities and correlation between these features within the set time range in order to compare the reference $f_{\mathrm{H}}^{i}$ and actual $f_{A}^{i}$ states of each heterogeneous area of the object for information support of end-users as regards agricultural monitoring and precision farming;

- provision of allocation results in the form of a heterogeneities' map.

The model is based on software and is built into the hardware-and-software complex of web-GIS for agricultural monitoring which belongs to Siberian Federal University $[9,10]$ and is available at the following Internet address: http://activemap.ikit.sfu-kras.ru. It co-functions with the software complex in offline mode and allows information support of end-users as regards evaluation of the integral state of Agricultural Lands.

\section{References}

[1] Raevich K V, Maglinets Yu A and Tsibulskii G M 2016 Intellectual Information System for evaluation of agricultural lands J. of Sib. Fed. Univ. Engin. \& Techn. 9 1025-1034

[2] Maglinets Yu A, Raevich K V and Tsibulskii G M 2017 Knowledge-based geoinformation technology for evaluation of agricultural lands Procedia Engineering 201 331-340

[3] Pelekis N 2004 Literature review of spatio-temporal database models $J$. The Knowledge Engineering Review 19(3) 235-274

[4] Jensen C S 2001 Introduction to Temporal Database Research CSCI Seminar 599

[5] Nandal R 2013 Spatio-Temporal Database and Its Models: A Review J. of Computer Engineering 91-100

[6] Dibihin K Yu 2009 Recognition of the elements of the underlying surface of agricultural landfill Multidisciplinary University as a Regional Center of Education and Science 587-591

[7] Brezhnev R V and Maglinets Yu A 2017 Information Support Technique for Solving Agricultural Land Monitoring Tasks Based on Earth Remote Sensing Data J. of Sib. Fed. Univ. Engin. \& Techn. 10(6) 819-827

[8] Rouse J W 1973 Monitoring vegetation systems in the Great Plains with ERTS ERTS Symposium NASA SP-351(I) 309-317

[9] Brezhnev R V, Maglinets Yu A, Maltsev E A, Perfilev S E, Sidorov A Yu, Tsibulskii G M and Shokol A S 2012 Software-and-technological infrastructure of information support for solving territorial administration tasks J. of Sib. Fed. Univ. Engin. \& Techn. 5(3) 340-352

[10] Maglinets Yu A, Tsibulskii G M and Noskov M V 2016 Modern trends to build up the regional ERS systems J. of Sib. Fed. Univ. Engin. \& Techn. 9(7) 1012-1018 